\title{
Detecting Coupled Domain Walls in Laboratory Experiments
}

\author{
Claudio Llinares* \\ Institute for Computational Cosmology, Department of Physics, Durham University, Durham DH1 3LE, United Kingdom \\ and Institute of Cosmology and Gravitation, University of Portsmouth, \\ Dennis Sciama Building, Portsmouth PO1 3FX, United Kingdom \\ Philippe Brax \\ Institut de Physique Théorique, Université Paris-Saclay, CEA, CNRS, F-91191 Gif-sur-Yvette Cedex, France
}

(Received 31 July 2018; revised manuscript received 3 December 2018; published 8 March 2019)

\begin{abstract}
The inherently unstable nature of domain walls makes their detection in laboratory experiments extremely challenging. We propose a method to stabilize domain walls inside a cavity. The method requires domain walls coupled to matter, a condition that is fulfilled by the symmetron model. We suggest two ways in which the walls could be detected once stabilized: studying the trajectories of ultracold neutrons either via the deflection angle of a neutron beam induced by the attraction towards the wall or through the time difference of these particles passing through the wall. We give realistic estimates for these effects and expect that they should be detectable experimentally.
\end{abstract}

DOI: 10.1103/PhysRevLett.122.091102

Introduction.-Domain walls are a particular class of topological defects that exist in many areas of physics $[1,2]$. Some domain walls appear in the gravity sector of alternative theories for gravity, where the scalar field which generates the walls couples to matter in a crucial way. Many theoretical works study the properties of topological defects in cosmology using both analytical [3-5] and numerical techniques [6-13]. Their interactions [14-18], gravitational effects [19-22], and emission of gravitational waves [23] are well investigated. However, efforts to detect them with cosmological observations have failed so far [24].

Here we focus on the detection in laboratory experiments. Unfortunately, it turns out that domain walls are unstable and do not last long enough to be detected with current experiments [25-28]. When the defects are coupled to matter, they minimize their energy in high density regions and move towards the walls of the experiment to disappear altogether [28-30]. On the other hand, domain walls can be stabilized inside a cavity by introducing a distribution of matter in the center of the experiment where the walls can attach themselves.

We focus on the symmetron model [31] although the effects that we obtain are not exclusive to this model. Our method only requires the domain walls to couple to matter. For instance, it is possible to add a disformal coupling to the conformal coupling without changing the existence of the domain walls attached to matter overdensities. Cosmological simulations with such a model were presented in Ref. [32], where topological defects were found. The same effects could also appear in vector-tensor theories in which the vector has a similar coupling as in the symmetron case (see. e.g., Ref. [33]) or in the generalized symmetron models discussed in Ref. [30].

We present ways of detecting domain walls too. These strategies involve using neutral particles' trajectories such as ultracold neutrons (UCNs) going across the wall or grazing the wall. UCNs have energies of the same order as the ones of the topological defects; hence, they are ideal for detection. Furthermore, there are already experiments where neutrons are cooled down to the desired low velocities. In both cases, either via the time difference compared to the situation with no wall or the deflection angle induced by the attraction towards the wall, we find that for particle beams with macroscopic velocities in the meters-per-second ballpark, and symmetron parameters compatible with previously studied experimental situations [34,35], such as atomic interferometry, the resulting effects should be detectable.

Symmetron model.-Stabilizing domain walls in cavity experiments requires the scalar field to be coupled to matter. We work with the symmetron model to present a realistic setup [31]. Predictions and constraints to the model exist in several contexts and spanning several orders of magnitude in scales including laboratory experiments [28,36], solar system scales [31,37], galaxy scales [38-41], galaxy clusters [42-45], cosmological scales [46-50], and variations of fundamental constants [51,52]. Furthermore, several symmetron $\mathrm{N}$-body cosmological codes exist [44,53-58].

The model is defined by the action

$$
S=\int \sqrt{-g}\left[R-\frac{1}{2} \nabla^{a} \phi \nabla_{a} \phi-V(\phi)\right] d^{4} x+S_{M}\left(\tilde{g}_{a b}, \psi\right),
$$


where the Einstein $g_{a b}$ and the Jordan $\tilde{g}_{a b}$ frame metrics are conformally related [i.e., $\tilde{g}_{a b}=A^{2}(\phi) g_{a b}$ ]. The potential and the conformal factor have the following forms:

$$
\begin{aligned}
& V(\phi)=-\frac{1}{2} \mu^{2} \phi^{2}+\frac{1}{4} \lambda \phi^{4}+V_{0}, \\
& A(\phi)=1+\frac{1}{2}\left(\frac{\phi}{M}\right)^{2},
\end{aligned}
$$

where $\mu$ and $M$ are mass scales which define the nature of the effective potential in the presence of matter, and $\lambda$ is a dimensionless constant. When coupled to nonrelativistic matter like the walls of a cavity in a laboratory experiment, the equation of motion of the symmetron field takes the following form when assuming a Minkowski background, as always realistic in an earthbound experimental context, and the quasistatic approximation for matter sources:

$$
\nabla^{2} \phi=\left(\frac{\rho}{M^{2}}-\mu^{2}\right) \phi+\lambda \phi^{3}
$$

The validity of this approximation in a cosmological context was tested in Refs. [56,57]. In a nutshell, the scalar field evolves in the following effective potential:

$$
V(\phi)_{\mathrm{eff}}=\frac{1}{2}\left(\frac{\rho}{M^{2}}-\mu^{2}\right) \phi^{2}+\frac{1}{4} \lambda \phi^{4}+V_{0} .
$$

Note that the potential is $Z_{2}$ symmetric and that symmetry breaking occurs at low energy. In regions of space when the density is smaller than $\rho_{\mathrm{SSB}}=\mu^{2} M^{2}$, the potential has two minima and, thus, can give rise to domain walls. In the opposite case, the $Z_{2}$ symmetry is restored and the scalar field is forced to oscillate around zero, screening the effects of its associated fifth force and allowing it to be consistent with Solar System observations [31]. This density dependence is also responsible for the unique properties of the domain walls which forces them to attach to high density regions $[29,30]$. This is analogous to ferromagnetism where domain walls interact with impurities (see, e.g., Ref. [59]). We now describe how this effect can be used to stabilize a wall inside vacuum cavities that are employed in usual experimental setups.

Stabilization of a coupled domain wall inside a cavity.As coupled domain walls are attracted to high density regions, we propose to stabilize them by introducing high density areas inside the vacuum chamber of the experiment. Thus the stabilizing process is the following: a phase transition is forced by generating vacuum inside the cavity (originally filled with a gas of density higher than $\rho_{\mathrm{SSB}}$ ). As the density is reduced, and during the resulting phase transition, the field can fall into one or the other minima of the effective potential and, thus, forms domain walls. This scenario can be realized in the laboratory by reducing the pressure of the gas inside the cavity to low values in a away akin to what would happen cosmologically during the cooling process of the Universe. In this case the phase transition would happen for cosmological symmetrons when the mass parameter $\mu$ induces a scalar force whose range is a fraction of the cosmological horizon. We focus on values of $\mu$ which are much larger and adapted to the modest size of experimental cavities (i.e., for the range $\mu^{-1}$ of the scalar interaction smaller than the size of the cavity). The resulting domain walls inside the cavity evolve searching for high density regions where they can minimize their energy. If any of the created walls happen to pass by the stabilizing element and the geometry of the stabilizer is correctly adapted to the model parameters, there is a nonnegligible probability that it will stay attached to it and become stable.

We tested this process by running 2D simulations with the code presented in Ref. [55] (already applied to coupled domain walls in Ref. [29]). As the simulations are 2D, the stabilizers are filaments with circular sections. Auxiliary filaments lying in the walls of the cavity may provide more control on the geometry of the final configuration. In these particular examples, the cavity is a $2 \mathrm{D}$ box whose size is $1 \mathrm{~m}$.

Figure 1 shows four stages of the experiment for two different configurations of the stabilizers. (i) Initial condition (left). The cavity is filled with gas and the scalar field is screened; i.e., its average value vanishes, with minimal perturbations around zero. (ii) Symmetry breaking and formation of a wall (middle left). Once the density of the gas inside the cavity falls below the density of symmetry breaking $\rho_{\mathrm{SSB}}$, the scalar field collapses to one or the other minima of the effective potential in different regions of space. At this stage, there is no guarantee that the resulting domain walls will survive. (iii) A survival domain wall is trapped by the filaments (middle right). The wall is now stable and will not collapse towards the walls of the cavity. Strong perturbations still exist in the wall, which does not have a straight configuration. (iv) Domain wall fully stabilized and ready to be detected (right). The perturbations in the wall are transferred to a background of scalar waves that travel through the whole cavity and are reflected on its sides. By doing this, the domain wall loses its kinetic energy and adopts an almost straight configuration, with minimal perturbations.

We estimated the probability of these events by running 1000 simulations with one stabilizing element in the center of the cavity. About $15 \%$ of these experiments gave rise to stable walls. The exact probability depends on the geometrical properties of the cavity and of the stabilizing elements. These simulations are inexpensive (i.e., fast) and thus will not constitute an issue when developing a real experiment.

The configurations of the filaments in Fig. 1 are the simplest ones for which we found stable domain walls. 


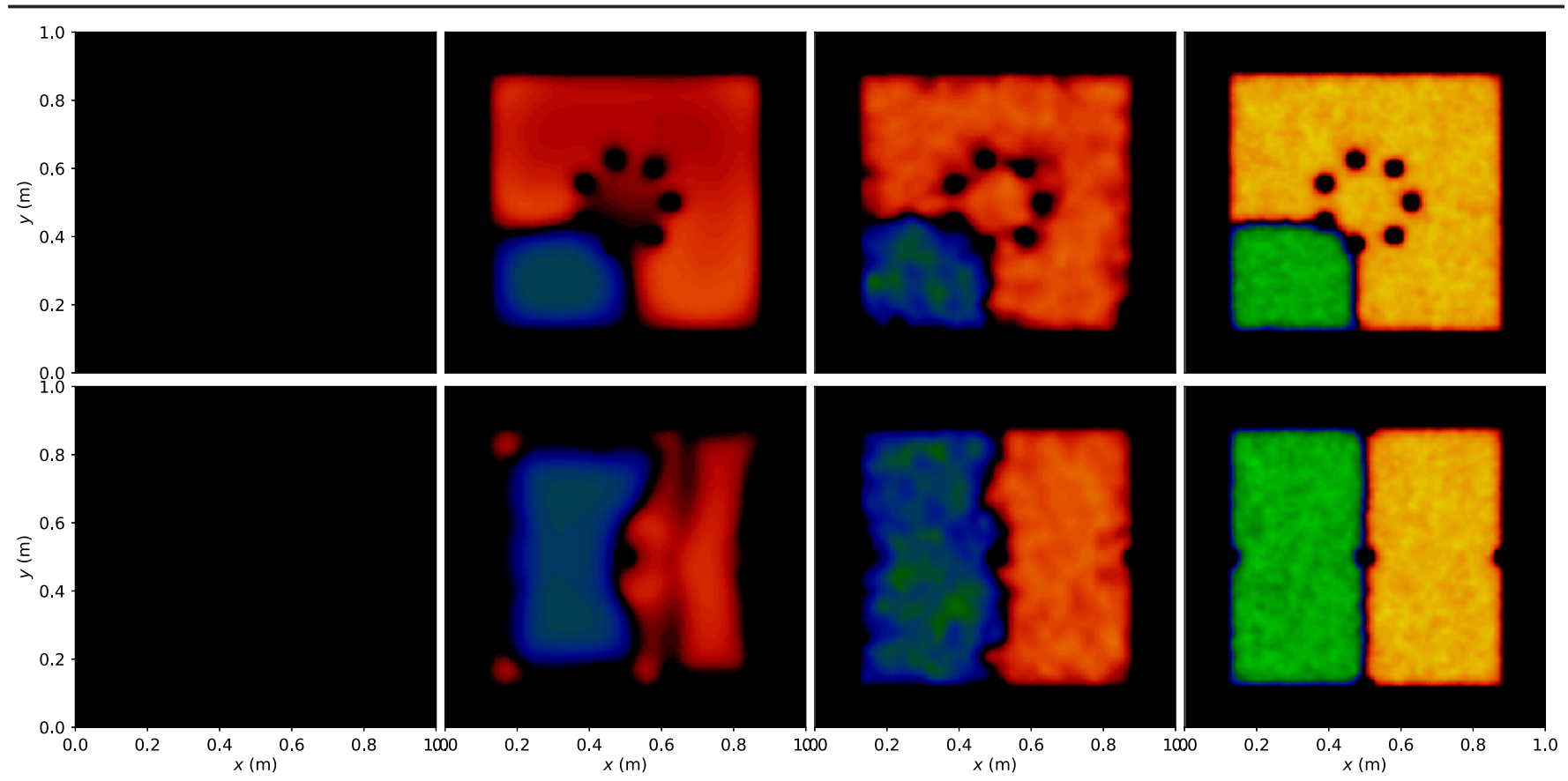

FIG. 1. Four stages of the generation of stable domain walls in a cavity. The colours correspond to the scalar field normalized to its vacuum value. The black regions around each panel are associated to the walls of the cavity, where the scalar field remains always screened. Each row corresponds to a different configuration of stabilizers while different columns show distinct phases in the evolution of the field. From left to right: (i) initial condition, (ii) symmetry breaking and formation of the wall, (iii) capture of the wall by filaments and (iv) wall fully stabilized.

However, this configuration is not unique. Differences in the arrangement of stabilizing elements affect the way the phase transition occurs and may increase the chances of forming a wall when releasing the gas [29]. Furthermore, moving sheets of material can be used to control the initial configuration of the walls after the phase transition occurred.

The upper row of Fig. 1 shows an example in which the wall is attached to the filaments and the walls of the cavity. In a different configuration, which also appears spontaneously in our simulations, the domain wall acquires the shape of a ring passing through all the filaments and detached from the walls of the cavity. The stability condition for such a configuration was studied in Refs. [29,60].

Detection of a domain wall inside a cavity.-We propose two different setups to detect stabilized domain walls both based on how the trajectories of slow particles, such as UCNs as presently used to search for chameleon fields [61-64], are affected. Neutrons can have energies of the order of $10^{-9} \mathrm{eV}$, which correspond to velocities of the order of $1 \mathrm{~m} / \mathrm{sec}$. We also assume values for the free parameters of the symmetron field compatible with the ones probed by atomic interferometry technique:

$$
\left(\mu_{0}, M_{0}, \lambda_{0}\right)=\left(2.4 \times 10^{-3} \mathrm{eV}, 10^{9} \mathrm{eV}, 0.1\right) .
$$

The value of $\mu$ is taken to be of the order of the dark energy scale. This is compatible with the fact that the quantum fluctuations due to the symmetron field of order $\mu^{4}$ could be at the origin of the acceleration of the expansion of the Universe. On the other hand, this large value of $\mu$ compared to the Hubble rate now $H_{0}$ implies that the scalar interaction mediated by the symmetron would have such a short range in vacuum, of the order of $0.1 \mathrm{~mm}$, that no fifth force manifestation of the symmetron would occur in the large scale structure of the Universe.

Trajectory of a massive particle around a domain wall: The trajectory of the neutrons in the vicinity of a domain wall satisfies Newton's law:

$$
\ddot{\mathbf{x}}=-c^{2} \frac{\nabla\left(\phi^{2}\right)}{2 M^{2}} .
$$

The effects of the gravitational field of Earth can be effaced by choosing the filaments to be vertical and studying the motion of particles in the horizontal plane. The solution in the $x$ direction, which we choose perpendicular to the wall and parallel to Earth's surface, can be obtained by taking into account that the Hamiltonian of the particle is conserved in each direction separately:

$$
H_{x}=\frac{\dot{x}^{2}}{2}+\frac{c^{2} \phi^{2}}{2 M^{2}}=\text { const. }
$$

Using the domain wall solution [1,2] of the field equation [Eq. (4)] and integrating once, we obtain the constraint 


$$
\int_{x_{0}}^{x(t)} \frac{d x}{\sqrt{1-a \tanh ^{2}(b x)}}=\sqrt{2 H_{x}}\left(t-t_{0}\right)
$$

where we have used the following definitions,

$$
\begin{array}{ll}
a \equiv \frac{c^{2} \phi_{0}^{2}}{2 H_{x} M^{2}}, & b \equiv \frac{1}{2 \lambda_{0}}, \\
\phi_{0}=\frac{\mu}{\sqrt{\lambda}}, & \lambda_{0}=\frac{\hbar c}{\sqrt{2} \mu},
\end{array}
$$

where $\phi_{0}=(\mu / \sqrt{\lambda})$ is the vacuum expectation value of the field and $\lambda_{0}=1 / m_{0}$ is its Compton wavelength in vacuum (see Ref. [29] for nonvacuum solutions). The numerical value of $\phi_{0}$ for our fiducial model defined by Eq. (6) is $7.5 \times 10^{-3} \mathrm{eV}$. We obtain the solution for the trajectory of a test particle,

$$
x(t)= \begin{cases}\frac{1}{b} \operatorname{asinh}\left(\frac{\sinh (\omega t+\alpha)}{\sqrt{1-a}}\right) & \text { if } a<1 \\ \frac{1}{b} \operatorname{asinh}\left(\frac{\sin (\omega t+\alpha)}{\sqrt{a-1}}\right) & \text { if } a>1,\end{cases}
$$

and its inverse,

$$
t(x)= \begin{cases}\frac{1}{\omega}\{\operatorname{arcsinh}[\sqrt{1-a} \sinh (b x)]-\alpha\} & \text { if } a<1 \\ \frac{1}{\omega}\{\arcsin [\sqrt{a-1} \sinh (b x)]-\alpha\} & \text { if } a>1 .\end{cases}
$$

There are two types of solutions depending on $a$, i.e., depending on the energy of the neutron in the $x$ direction. Figure 2 shows typical trajectories for initial conditions associated with values of $a$ greater (left) and smaller (right) than one. At low energy, the neutron oscillates around the domain wall due to the scalar attraction while at higher energy the neutron is refracted with a deflection depending on the strength of the scalar interaction. The characteristic

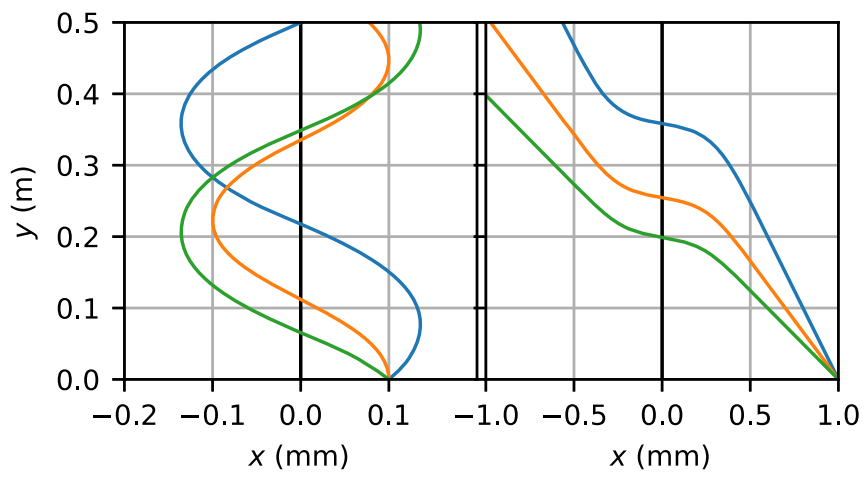

FIG. 2. Examples of trajectories of UCNs in the surroundings of a wall. The trajectories to the left and right correspond to values of $a$ greater than and smaller than one, respectively. Different curves correspond to different angles of the initial velocity. Note that the horizontal axes have different scale in different panels. energy separating the two types of behavior is given by $E_{x}=m_{N}\left(\mu^{2} / 2 \lambda M^{2}\right)$, where $m_{N}$ is the neutron mass. The solutions are governed by the frequencies and initial phases,

$$
\omega=b \sqrt{2 H_{x}|a-1|}=b v_{0}^{x}
$$

and

$$
\alpha= \begin{cases}\operatorname{arcsinh}\left[\sqrt{1-a} \sinh \left(b x_{0}\right)\right] & \text { if } a<1 \\ \arcsin \left[\sqrt{a-1} \sinh \left(b x_{0}\right)\right] & \text { if } a>1,\end{cases}
$$

respectively. Solutions are oscillatory for particles that are close enough to the wall and have a small transverse velocity.

Proposed experiments: The motion of a neutral test particle (e.g., a neutron) can be probed by two kinds of experiments. First, neutrons can be launched, for instance, from the bottom wall of the vacuum chamber moving upwards [i.e., with $\mathbf{v}=(\epsilon, 1) \mathrm{m} / \mathrm{sec}$. Small values of $\epsilon$ will give oscillatory trajectories. The period of these oscillations around the wall is shown in Fig. 3 for different values of the transverse velocity $\epsilon$. Since the periods are of the order of a few seconds and the total velocity of the neutrons is of the order of a few meters per second, the trajectory of the neutrons has a macroscopic displacement with respect to the trajectory that they would have in the absence of the domain wall and, thus, could be detected. There is a minimal period which corresponds to the limit $\left(x_{0}, v_{0}^{x}\right) \rightarrow(0,0)$ :

$$
P_{\min }=\sqrt{8} \pi \hbar \eta_{1}
$$

where we have $\eta_{1}=\sqrt{\lambda} M / \mu^{2}$. The numerical value of $P_{\min }$ for the fiducial model defined by Eq. (6) is $0.3 \mathrm{sec}$.

A different experiment could be realized by launching the neutrons in the transverse direction, for instance, moving from the left to the right walls of the cavity

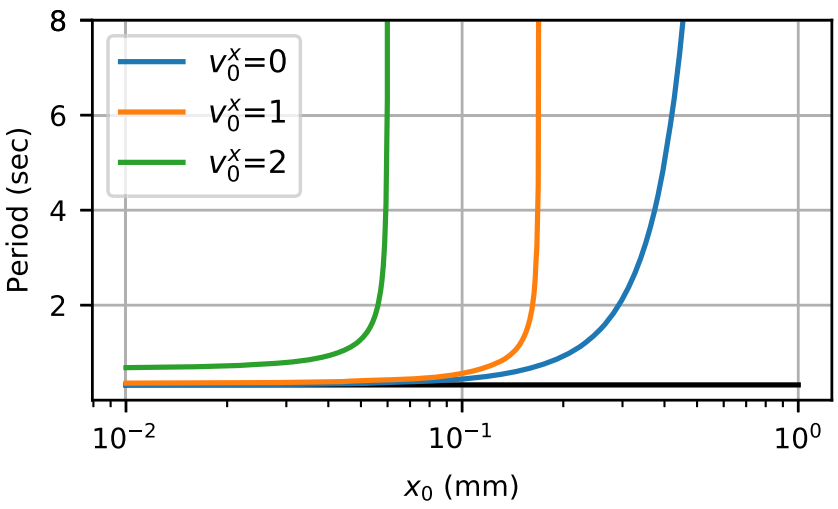

FIG. 3. Period of oscillations of a particle moving around a wall as a function of its initial position with respect to the wall. The three curves correspond to three different transverse velocities in meters per second. The horizontal line is the minimum period given in Eq. (16). 


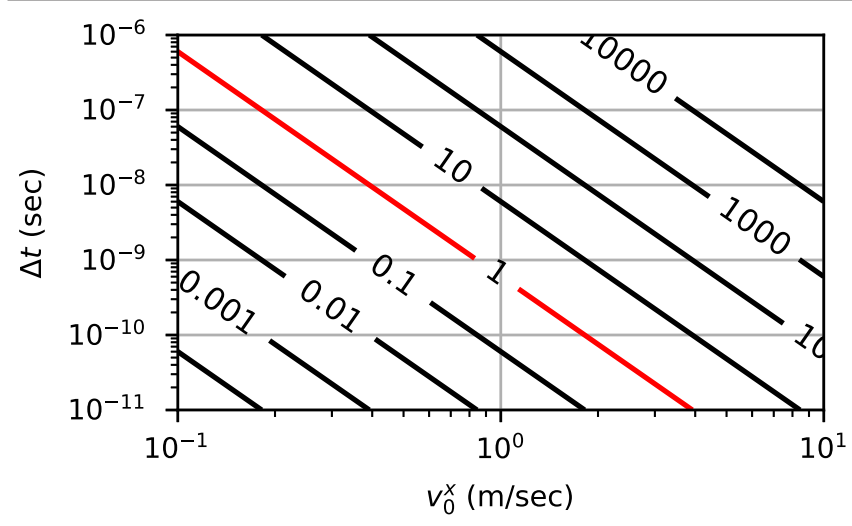

FIG. 4. Difference in arrival time of UCNs that travel across a wall in a direction perpendicular to it as a function of the initial velocity with respect to the wall and for different multiples of $\eta_{2}$ corresponding to Eq. (6).

[i.e., with $\mathbf{v}=(1,0) \mathrm{m} / \mathrm{sec}$ ]. These initial conditions are in the regime $a<1$ and, thus, the particles will move through the wall having only a small perturbation in their transverse velocity: particles will accelerate when approaching the wall and decelerate when moving away from it. Thus, there will be a difference in the arrival times between perturbed and unperturbed trajectories. This difference can be calculated by analyzing the asymptotic behavior of the trajectory in the initial and final positions of the particle:

$$
\Delta t=\sqrt{2} \frac{\hbar c^{3}}{\left(v_{0}^{x}\right)^{3}} \eta_{2},
$$

where we have defined $\eta_{2}=\mu /\left(M^{2} \lambda\right)$. Typical values of $\Delta t$ are shown in Fig. 4 for different multiples of the parameter $\eta_{2}$ associated to Eq. (6). This particular model in connection with UCNs traveling at $1 \mathrm{~m} / \mathrm{sec}$ (red curve) gives a difference in arrival times of the order of $10^{-9}$ sec.

We have estimated the range of parameters of the model which could be tested experimentally. First, we consider perturbations to UCNs' trajectories which could be detected when the period of oscillation around the wall would be of the order of the time they take to cross the cavity. Assuming a speed of $1 \mathrm{~m} / \mathrm{s}$ and a cavity of $1 \mathrm{~m}$, testable models are those with a period oscillation smaller than $1 \mathrm{sec}$. This constrains $\eta_{1}$. Similarly, we can assume a minimum measurable time delay (for instance of $10^{-9} \mathrm{sec}$ ) and obtain a constraint on $\eta_{2}$. Figure 5 shows the resulting exclusion regions. They cover regions of the parameter space that cannot be accessed otherwise [65].

Conclusions. - We have described the stabilization of symmetron domain walls in the vacuum chamber of an experiment when stabilizing filaments are present. The walls are the result of the symmetry breaking phase transition in the cavity when the gas density is lowered below the symmetry breaking scale. We have shown numerically how this process can be realized in an efficient way. Moreover, we have illustrated how to test for the presence of the walls in a cavity by calculating measurable effects that such a wall would have on UCNs' trajectories. For neutrons with velocities around $1 \mathrm{~m} / \mathrm{s}$, the deviation of their trajectories would be macroscopic for symmetron models with a scalar force range of the order of $0.1 \mathrm{~mm}$, as previously tested by atomic interferometry, and whose quantum fluctuations are in the right ballpark to generate the acceleration of the expansion of the Universe.
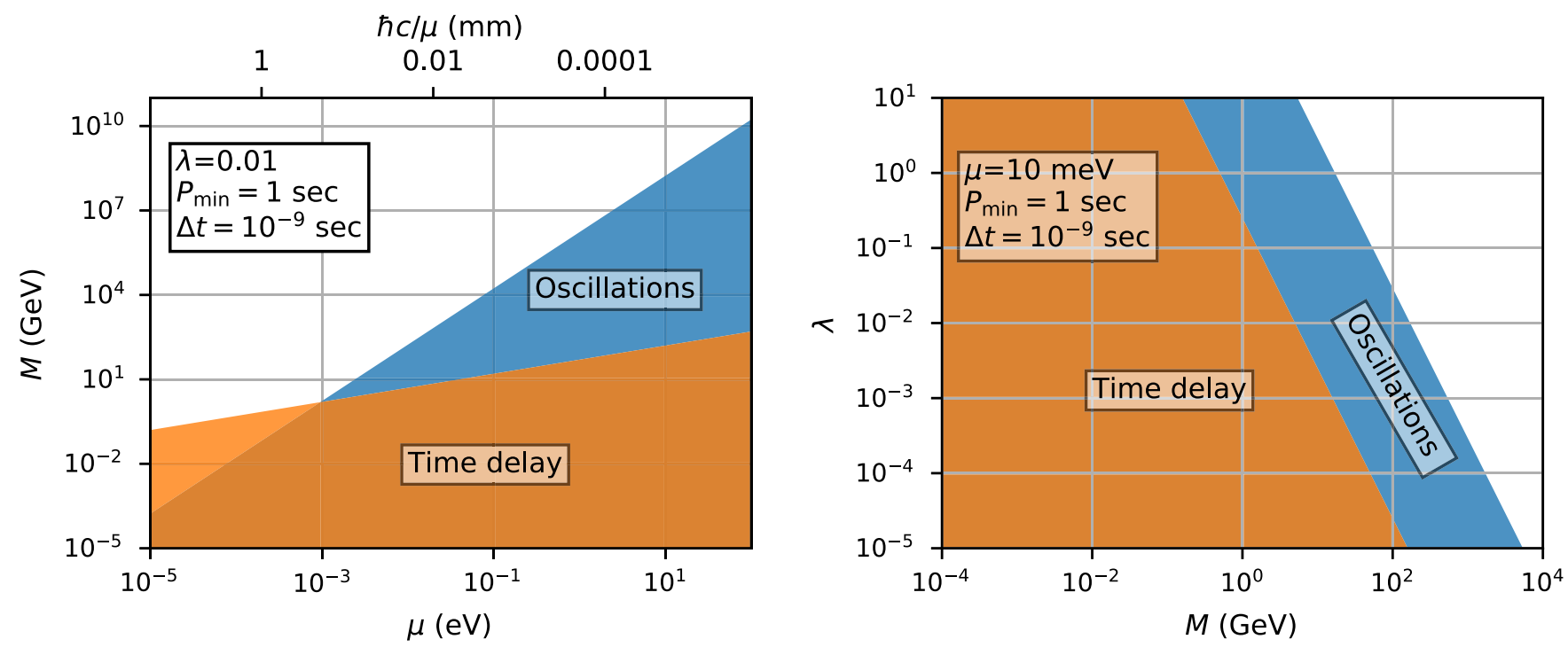

FIG. 5. Exclusion regions. Oscillations of UCNs that travel parallel to a wall (blue) and time delay of arrival of UCNs that travel perpendicular to a wall (orange). 
C. L. acknowledges support from STFC consolidated Grants No. ST/L00075X/1 and No. ST/P000541/1 and ERC Grant No. ERC-StG-716532-PUNCA. This work is supported in part by the EU Horizon 2020 research and innovation programme under the Marie-Sklodowska Grant No. 690575. This Letter is based upon work related to the COST Action CA15117 (CANTATA) supported by COST (European Cooperation in Science and Technology).

*claudio.llinares@port.ac.uk

[1] A. Vilenkin and E.P.S. Shellard, Cosmic Strings and Other Topological Defects (Cambridge University Press, Cambridge, UK, 2000).

[2] T. Vachaspati, Kinks and Domain Walls: An Introduction to Classical and Quantum Solitons (Cambridge University Press, Cambridge, England, 2006).

[3] M. Hindmarsh, Phys. Rev. Lett. 77, 4495 (1996).

[4] M. Hindmarsh, Phys. Rev. D 68, 043510 (2003).

[5] T. Vachaspati and A. Vilenkin, Phys. Rev. D 30, 2036 (1984).

[6] W. H. Press, B. S. Ryden, and D. N. Spergel, Astrophys. J. 347, 590 (1989).

[7] L. Kawano, Phys. Rev. D 41, 1013 (1990).

[8] D. Coulson, Z. Lalak, and B. Ovrut, Phys. Rev. D 53, 4237 (1996).

[9] S. E. Larsson, S. Sarkar, and P. L. White, Phys. Rev. D 55, 5129 (1997).

[10] T. Garagounis and M. Hindmarsh, Phys. Rev. D 68, 103506 (2003).

[11] P. P. Avelino, C. J. A. P. Martins, and J. C. R. E. Oliveira, Phys. Rev. D 72, 083506 (2005).

[12] A. M. M. Leite and C. J. A. P. Martins, Phys. Rev. D 84, 103523 (2011).

[13] A. M. M. Leite, C. J. A. P. Martins, and E. P. S. Shellard, Phys. Lett. B 718, 740 (2013).

[14] L. Pogosian and T. Vachaspati, Phys. Rev. D 62, 105005 (2000).

[15] L. Pogosian and T. Vachaspati, Phys. Rev. D 62, 123506 (2000).

[16] L. Pogosian, Phys. Rev. D 65, 065023 (2002).

[17] L. Pogosian and T. Vachaspati, Phys. Rev. D 67, 065012 (2003).

[18] N. D. Antunes, L. Pogosian, and T. Vachaspati, Phys. Rev. D 69, 043513 (2004).

[19] A. Vilenkin, Phys. Rev. D 23, 852 (1981).

[20] L. M. Widrow, Phys. Rev. D 39, 3571 (1989).

[21] K. Tomita, Phys. Lett. B 162, 287 (1985).

[22] J. Ipser and P. Sikivie, Phys. Rev. D 30, 712 (1984).

[23] T. Hiramatsu, M. Kawasaki, and K. Saikawa, J. Cosmol. Astropart. Phys. 02 (2014) 031.

[24] P. A. R. Ade et al. (Planck Collaboration), Astron. Astrophys. 571, A25 (2014).

[25] C. Burrage, E. J. Copeland, and E. A. Hinds, J. Cosmol. Astropart. Phys. 03 (2015) 042

[26] C. Burrage and E. J. Copeland, Contemp. Phys. 57, 164 (2016).

[27] C. Burrage, E. J. Copeland, and J. A. Stevenson, J. Cosmol. Astropart. Phys. 08 (2016) 070.
[28] C. Burrage, A. Kuribayashi-Coleman, J. Stevenson, and B. Thrussell, J. Cosmol. Astropart. Phys. 12 (2016) 041.

[29] C. Llinares and L. Pogosian, Phys. Rev. D 90, 124041 (2014).

[30] M. Peyravi, N. Riazi, and F. S. N. Lobo, Phys. Rev. D 95, 064047 (2017).

[31] K. Hinterbichler and J. Khoury, Phys. Rev. Lett. 104, 231301 (2010).

[32] R. Hagala, C. Llinares, and D. F. Mota, Astron. Astrophys. 585, A37 (2016).

[33] J. Beltrán Jiménez, A. L. Delvas Fróes, and D. F. Mota, Phys. Lett. B 725, 212 (2013).

[34] M. Jaffe, P. Haslinger, V. Xu, P. Hamilton, A. Upadhye, B. Elder, J. Khoury, and H. Mller, Nat. Phys. 13, 938 (2017).

[35] P. Brax and A.-C. Davis, Phys. Rev. D 94, 104069 (2016).

[36] A. Upadhye, Phys. Rev. Lett. 110, 031301 (2013).

[37] R. Hagala, C. Llinares, and D. F. Mota, Phys. Rev. Lett. 118, 101301 (2017).

[38] C. Burrage, E. J. Copeland, and P. Millington, Phys. Rev. D 95, 064050 (2017).

[39] C. Llinares, Mon. Not. R. Astron. Soc. 476, L29 (2018).

[40] C. A. J. O'Hare and C. Burrage, Phys. Rev. D 98, 064019 (2018).

[41] J. Clampitt, B. Jain, and J. Khoury, J. Cosmol. Astropart. Phys. 01 (2012) 030.

[42] M. B. Gronke, C. Llinares, and D. F. Mota, Astron. Astrophys. 562, A9 (2014).

[43] C. Llinares and D. F. Mota, Phys. Rev. Lett. 110, 151104 (2013).

[44] C. Llinares, D. F. Mota, and H. A. Winther, Astron. Astrophys. 562, A78 (2014).

[45] M. Gronke, C. Llinares, D. F. Mota, and H. A. Winther, Mon. Not. R. Astron. Soc. 449, 2837 (2015).

[46] K. Hinterbichler, J. Khoury, A. Levy, and A. Matas, Phys. Rev. D 84, 103521 (2011).

[47] P. Brax, C. van de Bruck, A.-C. Davis, B. Li, B. Schmauch, and D. J. Shaw, Phys. Rev. D 84, 123524 (2011).

[48] C. Llinares and N. McCullagh, Mon. Not. R. Astron. Soc. 472, L80 (2017).

[49] M. F. Ivarsen, P. Bull, C. Llinares, and D. Mota, Astron. Astrophys. 595, A40 (2016).

[50] L. Taddei, R. Catena, and M. Pietroni, Phys. Rev. D 89, 023523 (2014).

[51] M. F. Silva, H. A. Winther, D. F. Mota, and C. J. A. P. Martins, Phys. Rev. D 89, 024025 (2014).

[52] A. M. M. Pinho, M. Martinelli, and C. J. A. P. Martins, Phys. Lett. B 769, 491 (2017).

[53] A.-C. Davis, B. Li, D. F. Mota, and H. A. Winther, Astrophys. J. 748, 61 (2012).

[54] P. Brax, A.-C. Davis, B. Li, H. A. Winther, and G.-B. Zhao, J. Cosmol. Astropart. Phys. 10 (2012) 002.

[55] C. Llinares and D. F. Mota, Phys. Rev. Lett. 110, 161101 (2013).

[56] C. Llinares and D. F. Mota, Phys. Rev. D 89, 084023 (2014).

[57] H. A. Winther et al., Mon. Not. R. Astron. Soc. 454, 4208 (2015).

[58] R. Hagala, C. Llinares, and D. F. Mota, Astron. Astrophys. 585, A37 (2016).

[59] D. Jiles and D. L. Atherton, J. Magn. Magn. Mater. 61, 48 (1986). 
[60] J. A. Pearson, Phys. Rev. D 90, 125011 (2014).

[61] H. Lemmel, P. Brax, A. N. Ivanov, T. Jenke, G. Pignol, M. Pitschmann, T. Potocar, M. Wellenzohn, M. Zawisky, and H. Abele, Phys. Lett. B 743, 310 (2015).

[62] P. Brax, G. Pignol, and D. Roulier, Phys. Rev. D 88, 083004 (2013).
[63] P. Brax and G. Pignol, Phys. Rev. Lett. 107, 111301 (2011).

[64] T. Jenke, P. Geltenbort, H. Lemmel, and H. Abele, Nat. Phys. 7, 468 (2011).

[65] G. Cronenberg, P. Brax, H. Filter, P. Geltenbort, T. Jenke, G. Pignol, M. Pitschmann, M. Thalhammer, and H. Abele, Nat. Phys. 14, 1022 (2018). 